\title{
Clinicopathological Significance of Human Epidermal Growth Factor Receptor- 2(HER-2/Neu) Over-Expression in Gastric and Oesophageal Carcinomas of Upper Gastrointestinal Biopsies
}

\author{
Lynda DennisRodrigues* and Surekha B Hippargi
}

Dept. of Pathology, BLDE University's Shri BM Patil Medical College, Karnataka, INDIA

\begin{abstract}
Introduction: HER-2/neuplays a key role in the pathogenesis of gastric and oesophageal carcinomas and it's over expression has been documented in $6.8-34 \%$ of gastric carcinomas and $10-12.1 \%$ of oesophageal adenocarcinoma.Detecting the HER-2/neu status is a prerequisite for monoclonal antibody therapy.In this study, immunohistochemistry was used to detect HER-2/neu over- expression in gastric and oesophageal carcinomas.

Objective: To associate HER-2/neu over-expression withage, sex, type and grade of gastric and esophageal carcinomas in upper gastrointestinal (UGI) endoscopic biopsies.

Methods: HER-2/neu expression was investigated by immunohistochemistry on esophageal and gastric carcinomas ofUGI endoscopic biopsies received at our institution. Association between the expression of HER-2/neu andclinico-pathological parameters was statistically analysed.

Results: The association was not statistically significant between age, sexand grade of the tumour with HER-2/neu overexpression. HER-2/ neu overexpression was seen in $14.2 \%$ of gastric adenocarcinomas, $20 \%$ of esophageal adenocarcinomas and $4 \%$ of esophageal squamous cell carcinomas. Predominantly, intestinal type (9.5\%) of gastric carcinoma showed HER-2neu over-expression followed by diffuse type $(2.3 \%)$ and mixed type $(2.3 \%)$.
\end{abstract}

Conclusion: In view of increasing trend of UGI tract malignancies and associated poor survival of advanced carcinomas, assessing HER-2/ neu over-expression in gastric and esophageal carcinomas is helpful to decide the utility of adjuvant targeted chemotherapy.

Keywords: Adenocarcinoma, Endoscopic Biopsy, HER-2/neu, Immunohistochemistry.

\section{Introduction}

Gastric carcinoma is the second leading cause of mortality around the world ${ }^{[1]}$ whereas the incidence of esophageal carcinoma has increased more quickly than any other malignancy and is expected to rise in the coming decades. [2] Overall 5 year survival of gastric and esophageal carcinomas is between $5-10 \%$ due to the advanced stage at presentation..$^{[3]}$ With the advent of endoscopes and endoscopic biopsies, upper gastrointestinal endoscopy helps in early diagnosis and intervention.

Due to advanced stage of the disease during presentation, gastric and esophageal malignancies exhibit poor prognosis. Hence, the screening of high risk patients with endoscopic evaluation and biopsy as and when required plays a major role in detecting the cancer at an early stage.

Current targeted therapy for advanced gastric and esophageal carcinoma depends on the evaluation of target gene status. The overexpression of Human epidermal growth factor receptor 2 (HER-2/neu) has been documented in
$6.8-34 \%$ of gastric carcinomas including gastroesophageal junctional tumors. ${ }^{[4]}$ HER-2/neu positivity status plays a critical role in the development, progression and metastasis of malignancies such as breast cancer $\&$ gastric cancer. ${ }^{[2]}$

Detecting the HER-2/neu status in gastric and esophageal carcinoma is a prerequisite of monoclonal antibody therapy ${ }^{[1]}$ A combination of monoclonal antibody against HER-2/neu (Trastuzumab) with standard chemotherapy improves significant survival rates in patients with HER$2 /$ neu positive advanced gastric and esophageal cancers. ${ }^{[2]}$

Hence, overexpression of HER- 2/ neu plays an important role in cancer development and progression. However, this has not been documented extensively in literature. Under the light of this knowledge, the present study aims at investigating the frequency and the clinical significance of overexpression of HER- 2/ neu in gastric and esophageal carcinomas.

Objective of The Study: To correlate HER-2/neu overexpression with the type and grade of esophageal and 
gastric carcinomas in upper gastrointestinal endoscopic biopsy specimens.

\section{Methodology}

This is a prospective study. All UGI endoscopic biopsies received from the period $1^{\text {st }}$ December, 2015 to $30^{\text {th }}$ June, 2017 at the department of Pathology, of our hospital were subjected to the present study. The study included 107 gastric and esophageal UGI endoscopic biopsies that were diagnosed as carcinoma on histopathology. Poorly fixed specimens and biopsies with esophageal/gastric carcinomas that show association with histological changes of Barrett's esophagus were excluded from the study.

The number and size of the biopsy specimen was noted. Endoscopic biopsy tissues were fixed in $10 \%$ buffered formalin, which was followed by routine tissue processing and embedding in paraffin. Sections of 3-5 micron thickness were prepared and stained with Haematoxylin and Eosin.

Once a diagnosis of carcinoma was rendered, an extra section of 3 to $4 \mu \mathrm{m}$ was taken on pre-coated poly L lysine coated slides and further subjected to immunohistochemical staining with HER-2/neu.

\section{Results}

This study included 107 biopsies that were diagnosed as a malignant lesion on histopathology. Amongst them, 55 were from male patients and 52 belonged to female patients, M:F being 1.06:1. Majority of the cases (54.2\%) belonged to an age group above 60 years.

Out of all the malignancies that were diagnosed most of them were esophageal carcinomas followed by gastric carcinomas. GEJ accounted for only a minority of the cases. Squamous cell carcinoma was the predominant lesion in the esophagus, followed by adenocarcinoma. In GEJ, all cases were of adenocarcinoma. Stomach had maximum of adenocarcinomas followed by signet ring cell carcinoma. (Table 1 )

Lauren's classification was applied to gastric adenocarcinomas that revealed majority of cases to be diffuse type. (Figure 1)

IHC staining with HER-2/neu was performed. HER-2/ neu is a cytoplasmic membrane stain. Staining pattern was compared with control slides (known HER-2/neu positive breast cancer). HER-2/neu protein expression on the cell membrane was scored as described by Hofmann ${ }^{[5]}$ and colleagues for biopsy specimens, according to the criteria shown in Table 2.

Of the 42 cases that were diagnosed as adenocarcinomas, 06 (14.2\%) cases overexpressed HER- 2/neu, 4 of which were intestinal type (Figure 2) and one each of diffuse and mixed type. (Figure 3)

HER-2/neu analysis was also done on esophageal carcinomas. The frequency of HER-2/neu expression in these lesions was less than that seen in gastric carcinomas. Majority of squamous cell carcinomas were scored 0. Only 02 cases displayed $3+$ score (figure 4). Considering esophageal adenocarcinomas, 01/05 $(20 \%)$ case showed HER-2/neu positivity

Association of age of the patients, sex of the patients and grade of cancer with the HER-2/neu status (positive or negative) was done using Chi square test of independence. $\mathrm{P}$ value was not statistically significant $(>0.05)$

Table 1: Site-wise distribution of malignancies.

\begin{tabular}{|c|c|c|c|c|c|c|}
\hline Site & $\begin{array}{c}\text { Adeno- } \\
\text { carcinoma }\end{array}$ & $\begin{array}{c}\text { Signet ring cell } \\
\text { carcinoma }\end{array}$ & SCC & $\begin{array}{c}\text { Undifferentiated } \\
\text { carcinoma }\end{array}$ & $\begin{array}{c}\text { Adeno- } \\
\text { squamous } \\
\text { carcinoma }\end{array}$ & $\begin{array}{c}\text { Small round } \\
\text { blue cell tumor }\end{array}$ \\
\hline GE junction & 3 & 0 & 0 & 0 & 0 & 0 \\
\hline Esophagus & 5 & 1 & 50 & 5 & 2 & 1 \\
\hline Stomach & 31 & 4 & 0 & 4 & 0 & 0 \\
\hline
\end{tabular}

Table 2: HER-2/neu score in upper GI biopsy.

\begin{tabular}{|l|c|c|}
\hline Interpretation & Score & Interpretation \\
\hline No reactivity or membranous reactivity in $\leq 5$ cells. & 0 & Negative \\
\hline Faint/ barely perceptible membranous reactivity in $\geq 5$ cells & $1+$ & Negative \\
\hline Weak to moderate complete or basolateral membranous activity $\geq 5$ cells. & $2+$ & Equivocal \\
\hline Moderate to strong complete or basolateral membranous activity in $\geq 5$ cells. & $3+$ & Positive \\
\hline
\end{tabular}




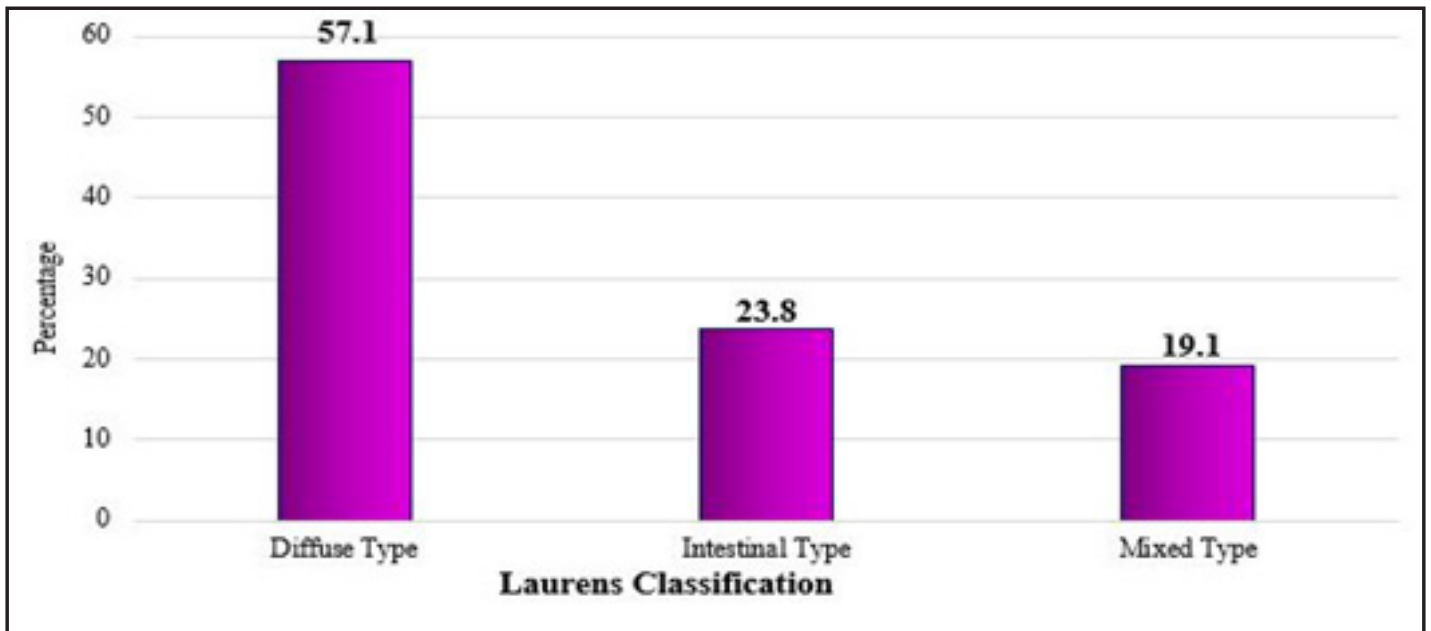

Fig. 1: Distribution of cases according to Laurens Classification.

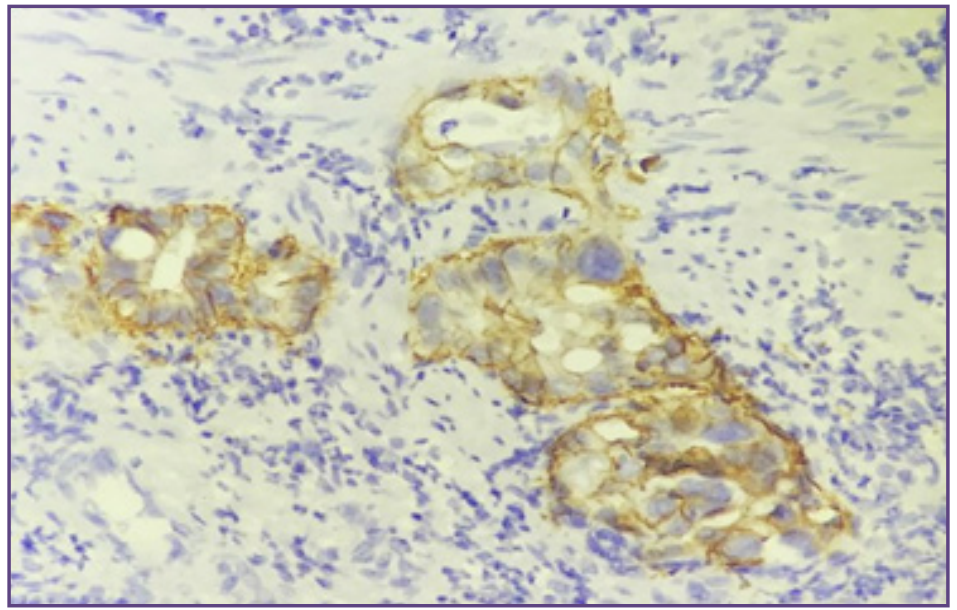

Fig. 2: IHC HER-2/ neu Score 3+ in intestinal type adenocarcinoma- Stomach. (IHC HER-2/ neu, 400x)

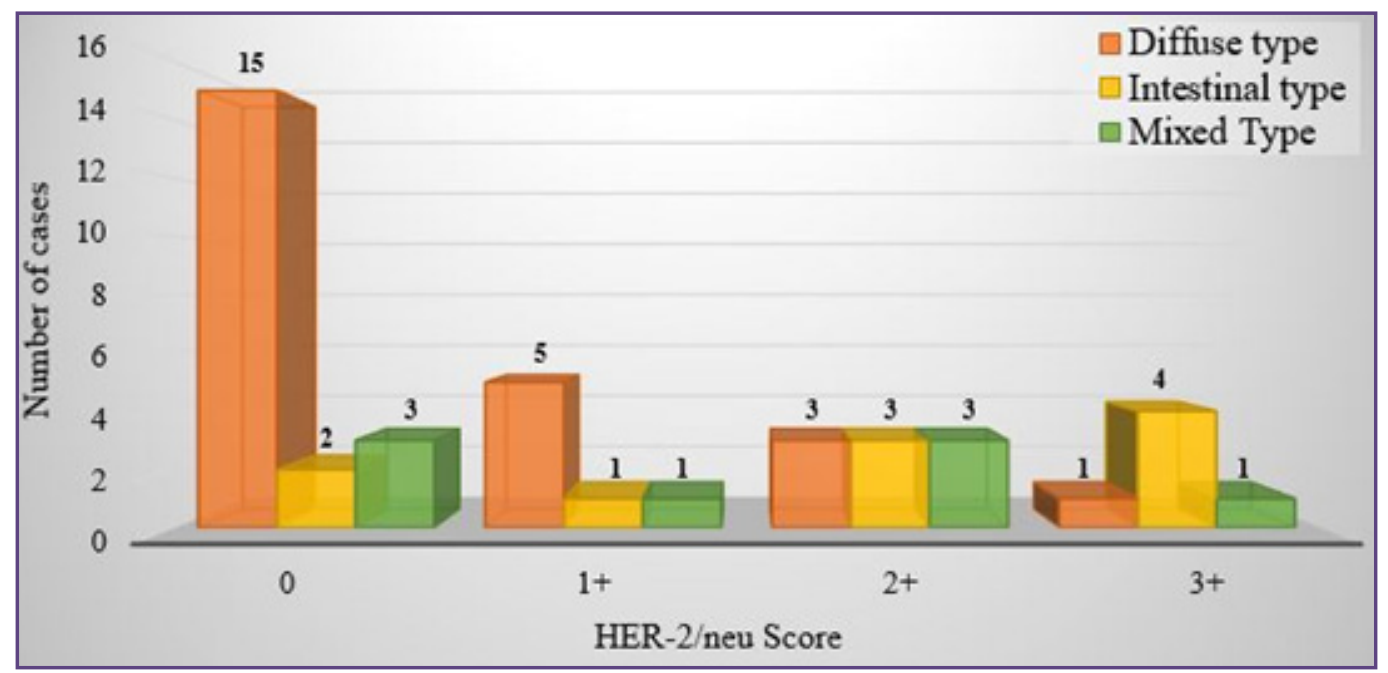

Fig. 3: HER-2/neu reactivity in gastric carcinomas

Annals of Pathology and Laboratory Medicine, Vol. 5, Issue 11, November, 2018 


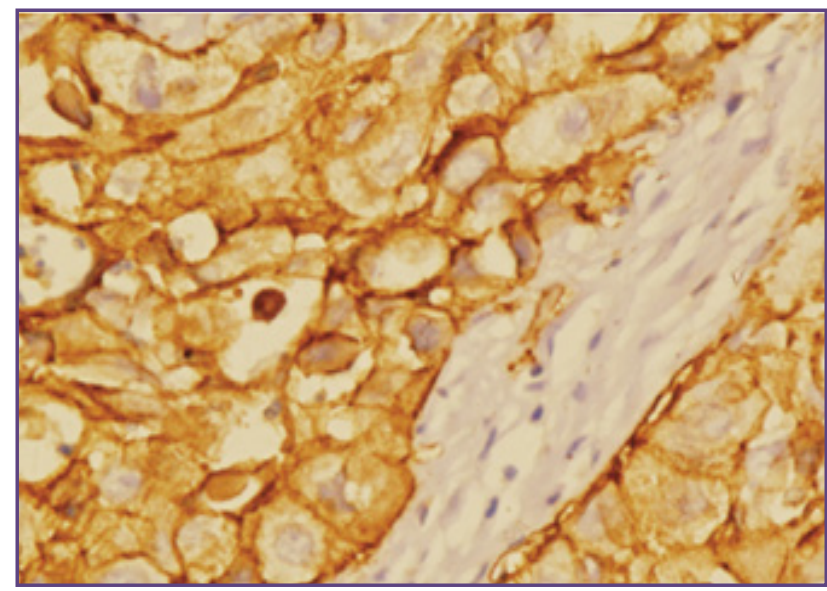

Fig. 4: IHC HER-2/ neu Score 3+ in well differentiated squamous cell carcinoma- Esophagus. (IHC HER-2/ neu, 400x).

\section{Discussion}

Endoscopic biopsies are common procedures done at the out-patient department to diagnose a variety of conditions. Such biopsies remain the mainstay for diagnosis of both non-neoplastic lesions and malignancies. Important information gained from biopsies can guide the surgeon to plan future management.

In the present prospective study, a total of 107 endoscopic biopsies that wre diagnosed as esophageal /gastric carcinoma on histopathology, were studied and analysed.

The age distribution was wide, ranging from 25 years to 85 years old. The mean age being 56.9 years. The majority of cases belonged to 60 to 70 years age group. Similar findings were seen in studies done by Qiu et al ${ }^{[6]}$ and Ahmadi et al. ${ }^{[7]}$

The number of males diagnosed as carcinoma on endoscopic biopsy was slightly more than females. The gender difference observed in upper gastrointestinal malignancies in the present study was consistent with studies done by Qui et $a l^{[6]}$ and Jawalkar et $a l^{[8]}$ High susceptibility in males can be due to personal habits of smoking and alcohol consumption; which are more common in males as stated by Krishnappa et al. ${ }^{[9]}$

In view of increasing trend of upper gastrointestinal malignancies and associated poor survival of advanced carcinomas, it is important to evaluate these carcinomas for expression of prognostic or theranostic molecular markers which can direct the use of specific targeted therapy. HER$2 /$ neu is a recent marker in the domain of gastric and esophageal carcinomas. Assessing HER-2/neu in these carcinomas not only predicts behaviour of carcinoma but also helps in deciding the chemotherapeutic drug to be used.

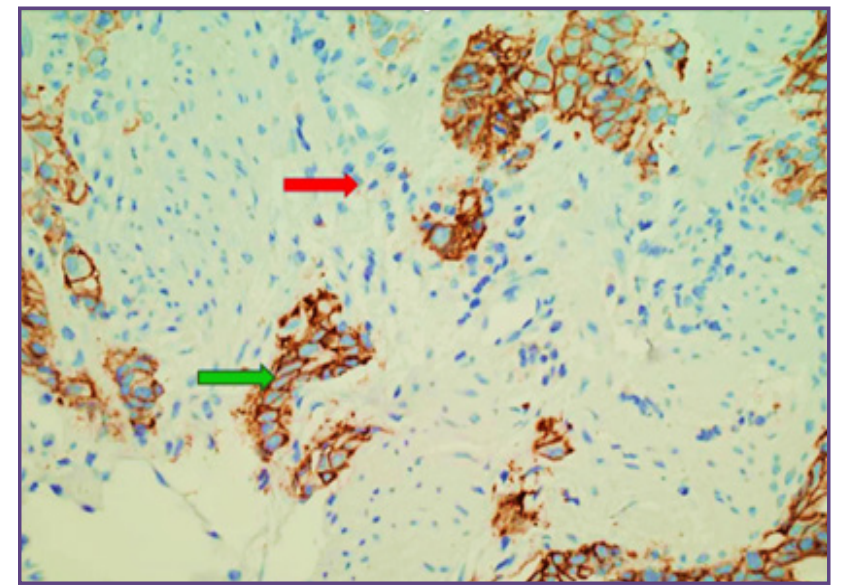

Fig. 5: Heterogenecity inHer-2 expression in mixed type gastric adenocarcinoma (Focal HER-2/neu over-expression in green and HER-2/neu negative in red arrow).

There is a wide variation in the HER-2 overexpression in gastric cancers. Reported rates of HER-2/neu expression in gastroesophageal cancer vary from the range of $2 \%$ to $45 \%{ }^{[10-13]}$ The largest data set of $>3800$ esophageal and gastric cancer samples found HER-2/neu protein positivity rate of $23 \%$. Hence, for clinical trial and treatment, it is very important to develop a standard HER- $2 /$ neu detection test to recruit eligible patients for Trastuzumab treatment.

Schoppmann et $a{ }^{[14]}$ in their study of HER-2/neu expression in esophageal carcinomas compared HER-2/ neu status using Hofmman ${ }^{[5]}$ scoring system (15.3\%) and Grabsch $^{[15]}$ scoring system (33.9\%). It showed a drastic difference between the results by the two methods. This observation reaffirms the importance of standardization of scoring as a prerequisite for selecting patients for targeted therapy. Hofmann ${ }^{5}$ scoring is similar to the guidelines given by EMA ${ }^{[16]}$ and ToGA ${ }^{[17]}$ and hence was used in the present study.

In the present study, HER-2/neu overexpression was seen in only $06(14.2 \%)$ cases of gastric carcinomas, of which $04(9.5 \%)$ were of intestinal type followed by one case each of diffuse and mixed type. Among the esophageal malignancies, $2 / 50(4 \%)$ of squamous cell carcinomas and $1 / 5(20 \%)$ of adenocarcinomas displayed HER-2/neu positivity. These results are consistent with similar studies done by Schoppmann et al ${ }^{[14]}$ and Reichelt et al. ${ }^{[18]}$

There is a wide variation in the overexpression of HER$2 /$ neu which can be explained by various factors such as, interpretation of data (considering only adenocarcinomas in the study) and the specimen (endoscopic biopsy/surgically resected) used for analysis. Even different geographic areas may exhibit variation in HER-2/neu expression. 
Overexpression of HER- 2/neu also depends on the type of carcinoma, whether it is squamous cell carcinoma or adenocarcinoma. It is more commonly associated with adenocarcinoma as studied by Schoppmann et al ${ }^{[19]}$ and Reichelt et al. ${ }^{[18]}$ These differences in expression of HER$2 /$ neu in different histologies of adenocarcinoma and squamous cell carcinoma suggest that HER-2/neu might have different prognostic implications in the two types. Allgayer et al[20] reported HER-2/neu overexpression to be an independent functional prognostic parameter for overall survival in gastric cancer.

In gastric adenocarcinomas, variation is seen in the subtypes as classified by Laurens classification. HER-2/neu overexpression is more commonly seen in intestinal type followed by diffuse and mixed type of adenocarcinomas. This observation was consistently seen in various studies by Moelans et al, ${ }^{[11]}$ Marx et al, ${ }^{[21]}$ Gravalos et al[ ${ }^{[22]}$ and Tanner et al. ${ }^{[23]}$ The findings of the present study are in concordance with the other studies.

The above mentioned variation in expression is because, intestinal and the diffuse types of adenocarcinomas have different molecular pathways of development and distinct genetic alterations. They also have different histological features. Lack of E-cadherin is inversely related to HER$2 /$ neu expression which is more commonly seen in the intestinal type of adenocarcinoma. ${ }^{[1]}$

The signet ring cells characteristically do not show HER$2 /$ neu expression. ${ }^{[12]}$ In the present study as well, signet ring cell carcinoma was HER-2/neu negative. Bakkelund et a ${ }^{[13]}$ proposed that signet ring cell carcinomas develop by gradual de-differentiation from ECL (enterochromaffin like) cells which indicate neuroendocrine origin of signet ring cells and hence they express markers like synaptophysin and chromogranin.

Silva et $a l^{[10]}$ ademonstrated HER-2/neu expression in both the components (isolated cells and glandular component) of mixed type of carcinoma. This suggests -

Common clonal origin of both the histological patterns and

HER-2/neu amplification is an early genetic alteration acquired before other epigenetic alterations associated with phenotypic divergence.

As per the study by Yan et al, ${ }^{[24]}$ among the mixed type of carcinomas, all the 3 cases displayed amplification in intestinal component, while one displayed amplification in diffuse component. In the present study, 1 case of mixed type of carcinomas had an intestinal component that exhibited HER-2/neu overexpression, whereas the diffuse component was negative.
In the present study HER-2/neu was overexpressed in $4 \%$ oesophageal squamous cell carcinoma and $20 \%$ of esophageal adenocarcinomas. Dreilch et $a l^{[25]}$ in their study concluded HER-2/neu overexpression was a poor prognostic factor for squamous cell carcinoma of the esophagus but had no effect on survival of patients with esophageal adenocarcinoma. Similarly, Akamatsu et al[26] stated HER-2/neu oncoprotein expression is associated with resistance to chemotherapy and radiotherapy in esophageal squamous cell carcinoma

Marked heterogeneity of HER-2/neu overexpression by gastric tumor tissue is documented in literature by various authors: Lee et al, ${ }^{[4]}$ Hofmann et al ${ }^{[14]}$ Ruschoff et al, ${ }^{[12]}$ Zhou et $a l^{[27]}$ and Tanner et al. ${ }^{[11]}$ On the contrary, only Marx et $a l^{[21]}$ in their study of 166 gastric cancers and 69 lymph node metastases concluded that HER-2/neu overexpression was highly homogenous in the primary tumor as well as in their metastases. In the present study, HER- 2/neu overexpression was heterogenous, and focal overexpression of HER- 2/neu, was seen. (Figure 5)

Majority of the upper GIT malignancies are treated with radiotherapy or chemotherapy. Dreilich et al[ ${ }^{[25]}$ stated that HER- 2/neu overexpression causes resistance to radiotherapy. Further, in a study by Akamatsu et al[26] esophageal carcinomas, HER-2/neu overexpression was associated with resistance to chemotherapy and radiotherapy.

The prognosis of HER-2/neu overexpression in gastric cancer and esophageal carcinomas is controversial. Also, association of HER-2/neu positive gastric cancer with clinicopathological features is not consistent.

In a study done by Wang et al ${ }^{[28]}$, it was observed that there was a higher frequency of HER-2/neu positivity in grade 2 carcinomas than in grade 3 because; many of the diffuse carcinomas are included in grade 3. Similar features were noted by Hu et al, ${ }^{[29]}$ Zhou et al, ${ }^{[27]}$ and Yan et al. ${ }^{[24]}$ In the present study, 107 gastric and esophageal carcinomas were studied and HER-2/neu overexpression was independent of grade of the carcinoma.

As stated by various authors [9, 4, 11, 22, 12, 25, 29] in their respective studies, in the present study as well, there was no correlation between the gender and the age of the patients of gastric and esophageal carcinoma with HER-2/ neu overexpression.

Dreilich et al ${ }^{[25]}$ showed that HER-2/neu 3+ score on IHC in esophageal squamous cell carcinomas is associated with poor survival whereas it had no effect on survival in patients with adenocarcinoma. Schoppmann et al ${ }^{[14]}$ studied HER$2 /$ neu expression in esophageal carcinomas and concluded 
that there is no association between the overall survival and squamous cell carcinoma or adenocarcinoma. However, Moleans et $a l^{[11]}$ described association of HER-2/neu overexpression with tumor invasion and tumor metastases in gastro-esophageal adenocarcinoma.

Yan et $a l^{[24]}$ described a significant inverse correlation between overall survival and HER-2/neu overexpression in intestinal type of gastric adenocarcinoma. Tanner et $a l^{[23]}$ and Zhou et $a l^{[27]}$ also concluded HER-2/neu as a poor prognostic indicator. In the present study, patient follow-up and survival was not studied.

Based on current analysis a clear trend towards a potential role for HER-2/neu as a negative prognostic factor in gastric and esophageal carcinomas was shown, suggesting that HER-2/neu overexpression or amplification is a molecular abnormality that might be linked to the development of gastric cancer.

\section{Conclusion}

HER-2/neu overexpression is seen predominantly in adenocarcinomas especially intestinal subtype and is associated with poor patient survival. A minor percentage of esophageal squamous cell carcinomas also express HER-2/neu oncoprotein. However, there is no association between the grades of the tumor, age and sex of the patient with HER-2/neu status. The level of HER-2/neu protein predicts well for the response of the carcinoma to monoclonal antibody, Trastuzumamb and is associated with poor prognosis. Evaluation of HER-2/neu as a routine diagnostic work up in UGI carcinomas may the useful. So IHC is recommended as the initial testing methodology.

\section{Acknowledgemnets:}

We would like to express our gratitude to Dr. Jakareddy, for his assistance and co-operation. We would also like to extend our special thanks to all the technical staff of the Department of pathology, Shr. BM Patil Medical College, Hospital and Research Centre for all the help and cooperation rendered during this study.

\section{Funding}

Department of Research and Development, BLDE University, Vijayapura,

\section{References}

1. Yan SY, Hu Y, Fan JG, Tao GQ, Lu YM, Cai X et al. Clinicopathologic significance of HER-2/neu protein expression and gene amplification in gastric carcinoma. World Journal of Gastroenterology 2011;17:1501-6.

2. Gowryshankar A, Nagaraja V, Eslick G.D. HER2 status in Barrett's esophagus \& esophageal cancer: A meta-analysis. Journal of Gastrointestinal Oncology 2014;5:25-35.
3. Amin A, Gilmour H, Graham L,Brown P, Terrace J, Crofts T.J. Gastric adenocarcinoma missed at endoscopy. J R Coll Surg Edinb 2002;47:681-4.

4. Lee S, Boer WB, Fermoyle S et al. Human epidermal growth factor receptor 2 testing in gastric carcinoma: Issues related to heterogeneity in biopsies and resections. Histopathology 2011;59:832-40.

5. Hofmann M, Stoss O, Shi D, et al. Assessment of a HER2 scoring system for gastric cancer: Results from a validation study. Histopathology 2008;52:797-805.

6. Qiu M, Zhou Y, Zhang X, Wang Z, Wang F, Shao J et al. Lauren classification combined with HER2 status is a better prognostic factor in Chinese gastric cancer patients. BMC Cancer 2014;14:823.

7. Ahmadi L, Kamkari S, Mokarram P.HER-2/neu and E-cadherin Expression and Microsatellite Instability in Gastric Dysplasia. Middle East Journal of Digestive Diseases 2011;3:20-7.

8. Jawalkar S, Arakeri S. Role of endoscopic biopsies in upper gastrointestinal diseases. Research Journal of Pharmaceutical, Biological and Chemical Sciences 2015;6: 977-83.

9. Rashmi K, Horakerappa MS, Karar A, Mangala G. International Journal of Medical Research \& Health Sciences 2013;2:418-24.

10. Silva JDB, Leitao D, Afonso L et al. Association of ERBB2 gene status with Histopathological parameters and diseasespecific survival in gastric carcinoma patients. B J Cancer 2009;00:487-93.

11. Moelans CB, Diest PJ, Milne ANA, et al. HER-2/neu Testing and Therapy in Gastroesophageal Adenocarcinoma. Pathology Research International 2011;ID 674182

12. Ruschoff J, Dietel M, Kreipe H. HER2 diagnostics in gastric cancer-guideline validation and development of standardized immunohistochemical testing. Virchows Arch.2010;457:299-307.

13. Junco JM, Jane H, Sawyer M B. Overview of Trastuzumab's Utility for Overview Adenocarcinoma: emerging therapeutic options and diagnostic challenges. Connection 2010;15:47-51.

14. Schoppmann SF, Jesch B, Friedrich J et al. Expression of HER-2 in Carcinomas of the Esophagus. Am J SurgPathol 2010;34:1868-73.

15. Grabsch H, Sivkumar S, Gray S et al. HER2 expression in Gastric Cancer: Rare, heterogenous and of no prognostic value- conclusion from 924 cases of two independent series. Cell Oncol 2010;32:57-65.

16. European Medicines Agency. Assessment Report for Herceptin. Doc. Ref. No. EMA/842364/2009.

17. Bang YJ, Van CE, Feyereislova A, at al. Trastuzumab in combination with chemotherapy versus chemotherapy alone for treatment of HER-2/neu positive advanced gastric or 
gastroesophageal junction cancer (ToGA): A phase 3, open label, randomized control trial. Lancet 2010;376:687-97.

18. Reichelt U, Duesedau P, Tsourlakis MC et al. Frequent homogenous HER-2 amplification in primary and metastatic adenocarcinoma of the esophagus. Modern Pathology 2007;20:120-9.

19. Schoppmann SF, Jesh B, Zacheri J et al. HER-2 status in primary oesophageal cancer, lymph nodes and distant metastases. British Journal of Surgery 2011;98:1408-13.

20. Allgayer H, Babic R, Gruetzner KU et al. C-erb-2 is of independent prognostic relevance in gastric cancer and is associated with the expressison of tumor associated protease systems. J Clin Oncol 2000;18:2201-9.

21. Gravlos C, Jimeno A. HER2 in gastric cancer: A new prognostic factor and a novel therapeutic target. Annals of oncology 2008;19:1523-9.

22. Marx AH, Tharun L, Muth J et al. HER-2 amplification is highly homogenous in gastric cancer. Human Pathology 2009;40:769-77.

23. Tanner M, Hollmen M, Junttila TT et al. Amplification of HER-2 in gastric carcinoma: Association with Topoisomerase II $1 \alpha$ gene amplification, intestinal type, poor prognosis and sensitivity to Trastuzumab. Annals of Oncology 2005;16:273-8.
24. Principles, Pitfalls and Standardization. In: Zorab R. (Eds). Diagnostic Immunohistochemistry. 1st ed. Philadelphia: Chruchill Livingstone;2002. p.3-43.

25. Dreilich M, Wangers A, Brattstrom D et al. Her-2 overexpression $(3+)$ in patients with squamous cell carcinoma correlates with poor survival. Disorders of the Esophagus 2006;19:224-31.

26. Akamatsu $\mathrm{M}$, Matsumoto $\mathrm{T}$, Oka $\mathrm{K}$ et al. c-erb B-2 oncoprotein expression related to chemoradioresistance in esophageal squamous cell carcinoma. Int J Radiot Oncol Bio Phys 2003;57:1323-7.

27. Zhou Z, Hick DG. Oncogene and Cancer- From Bench to Clinic [internet].USA: 2013. HER2 Amplification or Overexpression in Upper GI Tract and Breast Cancer with Clinical Diagnosis and Treatment.[cited 2017 March 24]. Available from: http://dx.doi.org/10.5772/53921

28. Wang S, Zheng G, Chen L et al. Effect of HER-2/neu expression on prognosis of gastric cancer: A Meta-analysis. Asian Pacific J Cancer Preview 2011;12:1417- 23.

29. Hu Y, Bandla S, Godfrey TE, et al. HER2 amplification, overexpression and score criteria in esophageal adenocarcinoma. Modern Pathology 2011;24:899-907.

*Corresponding author:

Dr. Lynda DennisRodrigues, Dept. of Pathology, BLDE University's Shri BM Patil Medical College, Karnataka, INDIA

Email: lyndacrodrigues@gmail.com

Financial or other Competing Interests: None. 